\title{
Monitoramento da qualidade do ar no campus Três Poços em termos de partículas totais em suspensão.
}

\section{Monitoring of air quality on Três Poços campus in terms of total suspended particles.}

Artigo

Original

\author{
Derek Gomes ${ }^{1}$ \\ Fabrício Barcelos Sá ${ }^{1}$ \\ Mateus Nascimento Mariano ${ }^{1}$ \\ Ramon Castro Vianna ${ }^{1}$ \\ Anderson Gomes ${ }^{2}$ \\ Marcus Vinicius F. de Araujo ${ }^{3}$ \\ Rosana A. Ravaglia Soares ${ }^{4}$
}

Original

Paper

\section{Palavras-chave: \\ Partículas totais em suspensão}

Qualidade do ar Agv pts

\section{Resumo}

Segundo o INEA, Poeiras em suspensão no ar diminuem a capacidade de remoção das partículas pelo sistema respiratório e potencializam os efeitos dos gases. O objetivo desta pesquisa é monitorar a qualidade do ar no campus Três Poços do UniFoa, em termos de partículas totais em suspensão na bacia aérea local. Os resultados permitem um acompanhamento da qualidade do ar em todo o campus para um diagnóstico. Foram consideramos dados da estação meteorológica em Volta Redonda - RJ. O monitoramento foi feito pelo Amostrador de Grande Volume para Partículas Totais em Suspensão AGV PTS, o qual determina concentrações de partículas totais em suspensão (PTS). O equipamento foi calibrado por um calibrador portátil aferido pela empresa ENERGÉTICA em 25 / 05 / 2009 com prazo de validade 25 / 05 / 2010. O resultado da pesquisa é importante para todos os frequentadores do campus Três Poços, pois a qualidade do ar afeta o Meio Ambiente, a saúde e a qualidade de vida dos mesmos.

\begin{abstract}
According to INEA, Dust in the air decreases the ability to remove particles from the respiratory system and enhance the effects of the gases. The purpose of this research is to monitor air quality on campus UniFoa Three Wells in terms of total suspended particulates in the air basin site. The results allow monitoring of air quality throughout the campus for a diagnosis. We consider data from the meteorological station in Volta Redonda - RJ. The monitoring was done by High Volume Sampler for Total Suspended Particulate AGV PTS determining concentrations of total suspended particles (TSP). The equipment was calibrated by a portable calibrator measured by the energy company on 25 / 05 / 2009 with expiry date 25 / 05 / 2010. The search result is important for all visitors to the campus three wells, because the air quality affects the environment, health and quality of their lives.
\end{abstract}

Key words:

Total suspended particulates

Air quality

Agv pts

\footnotetext{
${ }^{1}$ Discente do curso Engenharia Ambiental - UniFOA (Centro Universitário de Volta Redonda), Volta Redonda/RJ, Brasil.

${ }^{3}$ Graduado em Engenharia Química - Graduação em Teologia - Especialização em Docência Superior - Mestrado em Plaejamento Energético ${ }^{4}$ Graduada em Engenharia Metalúrgica - Mestrado em Engenharia Metalúrgica e de Materiais - Mestrado em Engenharia Metalurgica e de
} Materiais 


\section{Introdução}

A poluição do ar pode ser definida como:

“Alteração das propriedades físicas, químicas ou biológicas normais da atmosfera que possa causar danos reais ou potenciais à saúde humana, à flora, à fauna, aos ecossistemas em geral, aos materiais e à propriedade, ou prejudicar o pleno uso e gozo da propriedade ou afetarasatividadesnormaisdapopulação ou o seu bem estar" (Hasegawa, 2001).

\section{Objetivo}

O projeto tem como proposta o monitoramento da qualidade do ar no Campus Três Poços, situado no município de Volta Redonda.

\section{Impactos Relacionados a Partículas Totais em Suspensão}

As partículas em suspensão no ar afetam a capacidade do sistema respiratório se depositando nas paredes dos pulmões. Quando muito finas, as partículas conseguem uma maior penetração no aparelho respiratório, aumentando a possibilidade de trazer danos à saúde.

A junção de fuligem (C) e Hidrocarbonetos Aromáticos Policíclicos, quando inaladas pelo homem, pode causar sérios problemas à saúde, prejudicando a qualidade de vida de todos os envolvidos.

\section{Aplicações do HI-VOL}

O Amostrador de Grande Volume para Material Particulado determina as concentrações (em $\mu \mathrm{g}$ / $\mathrm{m}^{3}$ ) de partículas totais em suspensão (PTS) no ar ambiente externo e interno, monitorando ambientes industriais (higiene industrial), incluindo coleta de amostras de materiais altamente tóxicos, análise de poluentes orgânicos, presença de metais ( $\mathrm{Si}, \mathrm{Ca}, \mathrm{Na}, \mathrm{Pb}, \mathrm{Zn}$ e outros) e medidas da concentração de radioatividade em poeira em suspensão. Um exemplo é uma mina com minério contendo urânio/tório como materiais secundários.

O HI-VOL também é usado no estudo de impactos ambientais para determinar níveis preexistentes da qualidade do ar e monitoramento de emissões fugitivas de processos industriais quando não é possível utilização de amostradores em chaminés/dutos.

\section{Procedimentos de Operação}

Primeiramente foi feito um estudo técnico do HI-VOL, aprendendo a operar-lo, conhecendo-se o manual do aparelho.

Para dar início às análises, foi necessário fazer a calibração do equipamento.

A seguir, objetivando a análise da qualidade do ar no Campus Três Poços, a fim de evitar interferências físicas de outros prédios ou outras edificações do campus, escolhemos o prédio da medicina para a instalação do Hi-VOL .

Localização ( $\square: 22^{\circ} 29^{\prime} 58,38^{\prime \prime}$; ; $\square: 44^{\circ} 02^{\prime} 08,66^{\prime \prime O}$ ), fonte: WGS84

Programamos o equipamento para funcionar em dias de maior e menor fluxo da população flutuante no Campus, o que nos deu a resposta da origem da maior quantidade de particulados.

\section{Cálculos}

Após cada dia de coleta, o material era pesado e sua concentração calculada.

Para a determinação da concentração de amostragem foi usada a seguinte fórmula:

$$
\begin{aligned}
& \mathrm{C}=\mathrm{Q} \cdot \mathrm{t}=\operatorname{Var} \\
& \mathrm{C}=\operatorname{Var} . \Delta \mathrm{P}
\end{aligned}
$$

Sendo:

Q - vazão de sucção (m3/s)

$\mathrm{t}$ - tempo de funcionamento do aparelho (24h)

Var - volume de ar succionado (m3)

$\Delta \mathrm{P}$ - Pfiltro final - Pfiltro inicial $(\mu \mathrm{g} / \mathrm{m} 3)$

Para a determinação da média geométrica das concentrações dos dias de amostragem foi usada a seguinte fórmula:

Média Geométrica $(17 / 06$ a 25/09) $=(154,1$ x 79,7 x $82,8 \times 40,4 \times 41,4 \times 34,5 \times 36,7 \times 36,2 \times 112,7 \times 91,5$ $\mathrm{x} 70,1 \times 107,9 \times 100,3) 1 / 13$ 
Para a determinação das concentrações médias diárias:

Concentrações médias $=\sum$ concentrações $\mathrm{n}^{\circ}$ de amostras

\section{Dados Meteorológicos}

Partindo-se do princípio que partículas em suspensão podem percorrer uma grande distância quando influenciadas por ventos incidentes, torna-se indispensável a posse de informações meteorológicas da região.

Como a CSN (Companhia Siderúrgica Nacional) é uma empresa geradora de partículas em suspensão, a consulta da direção dos ventos relativos à empresa e ao Campus Três Poços era imprescindível para o conhecimento da origem da maior influência nas concentrações altas de particulados no ambiente aéreo do Campus Três Poços.

Em função dos dados meteorológicos coletados pela Estação Meteorológica da CSN (tabela 01), foi gerada uma Rosa dos Ventos (imagem 01) utilizando-se o programa WRPLOT View.

Com a velocidade dos ventos classificados em ordens de calmo a forte e a quantificação da repetição das velocidades mais próximas, foi gerado um gráfico com a frequência dos ventos (Imagem 02) através do WRPLOT View.

Ao se analisar a Rosa dos Ventos e a imagem de localização da CSN e do Campus da UniFOA (imagem 03), é possível ter uma visão mais clara da influência dos ventos no Campus

\begin{tabular}{|c|c|c|c|c|c|c|c|c|c|c|c|c|}
\hline \multirow[t]{7}{*}{ Data de Mediçăo } & \multicolumn{12}{|c|}{ TABELA 01} \\
\hline & \multicolumn{12}{|c|}{ Estacalo Meteorologica } \\
\hline & \multicolumn{12}{|c|}{ Meteorologia } \\
\hline & \multicolumn{12}{|c|}{ Clima e Meteorologia } \\
\hline & \multirow{3}{*}{\multicolumn{2}{|c|}{$\begin{array}{l}\text { Pressao } \\
\text { Atmostérica } \\
\text { Frequetncla } \\
\text { Horiria com } \\
\text { amostra de } 1 \text { Hora } \\
\text { a } 0.0 \mathrm{~m} \\
\text { Rotina }\end{array}$}} & \multirow{3}{*}{\multicolumn{2}{|c|}{$\begin{array}{l}\text { Umidade Relativa } \\
\text { Frequencia } \\
\text { Horária com } \\
\text { amostra de } 1 \\
\text { Hora a } 0.0 \mathrm{~m} \\
\text { Rotina }\end{array}$}} & \multirow{2}{*}{\multicolumn{2}{|c|}{$\begin{array}{l}\text { Velocidade Escalar } \\
\text { do Vento } \\
\text { Frequencia Hordiria } \\
\text { com amostra de } 1 \\
\text { Hora a } 0.0 \mathrm{~m}\end{array}$}} & \multirow{2}{*}{\multicolumn{2}{|c|}{$\begin{array}{l}\text { Diresalo Escalar } \\
\text { do Vento } \\
\text { Freqoencia } \\
\text { Horária com } \\
\text { amcstra de } 1 \\
\text { Hora a } 0.0 \mathrm{~m}\end{array}$}} & \multicolumn{2}{|c|}{ Temperatura } & \multicolumn{2}{|c|}{$\begin{array}{l}\text { Precipitaça } \\
\text { Pluviometrica }\end{array}$} \\
\hline & & & & & & & & & $\begin{array}{l}\text { Frequencia } \\
\text { Horaria } \\
\text { amostra } \\
\text { Hora a } 0,0\end{array}$ & $\begin{array}{l}\text { com } \\
\text { de } 1\end{array}$ & $\begin{array}{l}\text { Frequencia } \\
\text { com amostr } \\
\text { Hora a } 0.0 \mathrm{~m}\end{array}$ & $\begin{array}{l}\text { Horánia } \\
\text { de } 1\end{array}$ \\
\hline & & & & & Rotina & & Rotina & & Rotina & & Rotina & \\
\hline Data & $\begin{array}{l}\text { Valor } \\
\text { [mbar] }\end{array}$ & Flag & Valor [\%] & Flag & Valor [m/s] & Flag & Valor ["] & Flag & Valor $\left[{ }^{\circ} \mathrm{C}\right]$ & Flag & Valor $[\mathrm{mm}]$ & Flag \\
\hline $17 / 06 / 200900-30$ & 725 & & 99.9 & & 0 & & 0 & & 15,8 & & 0 & \\
\hline 17/06/2009 01:30 & 725 & & 99.9 & & 0 & & 0 & & 15,5 & & 0 & \\
\hline $17 / 06 / 20090230$ & 725 & & 99.9 & & 0 & & 0 & & 15,1 & & 0 & \\
\hline $17 / 06 / 200903.30$ & 725 & & 99.9 & & 0 & & 0 & & 14.5 & & 0 & \\
\hline $17 / 06 / 200904: 30$ & 725 & & 99,9 & & 0 & & 0 & & 14,3 & & 0 & \\
\hline $17 / 06 / 200905.30$ & 725 & & 99,9 & & 0 & & 0 & & 13,9 & & 0 & \\
\hline $17 / 06 / 200906.30$ & 725 & & 99,9 & & 0 & & 0 & & 13.5 & & 0,2 & \\
\hline $17 / 06 / 200907: 30$ & 726 & & 99,9 & & 0 & & 0 & & 13,9 & & 0 & \\
\hline $17 / 06 / 200908.30$ & 726 & & 99,9 & & 0 & & 0 & & 14,8 & & 0 & \\
\hline $17 / 06 / 200902.30$ & 727 & & 99,9 & & 0 & & 0 & & 16,7 & & 0 & \\
\hline $17 / 06 / 200910-30$ & 728 & & 91,3 & & 0 & & 296 & & 20,1 & & 0 & \\
\hline $17 / 06 / 200911: 30$ & 728 & & 73,2 & & 0 & & 0 & & 23,9 & & 0 & \\
\hline $17 / 08 / 200912: 30$ & 727 & & 64.6 & & 0 & & 286 & & 26.6 & & 0 & \\
\hline $17 / 06 / 200913.30$ & 727 & & 49,1 & & 0 & & 239 & & 29,7 & & 0 & \\
\hline $17 / 06 / 200914: 30$ & 727 & & 49,4 & & 0 & & 279 & & 29,3 & & 0 & \\
\hline $17 / 06 / 200915: 30$ & 726 & & 48.2 & & 0 & & 153 & & 29.2 & & 0 & \\
\hline $17 / 06 / 200916-30$ & 726 & & 81,1 & & 3,5 & & 123 & & 22,6 & & 0,2 & \\
\hline $17 / 06 / 200917: 30$ & 726 & & 87,8 & & 2,9 & & 127 & & 20.9 & & 0 & \\
\hline $17 / 06 / 200918: 30$ & 727 & & 92,3 & & 2,3 & & 105 & & 19,8 & & 0 & \\
\hline $17 / 06 / 200919 \cdot 30$ & 727 & & 94,8 & & 2,1 & & 99 & & 19,3 & & 0 & \\
\hline $17 / 06 / 200920-30$ & 728 & & 96,1 & & 1,3 & & 102 & & 19 & & 0 & \\
\hline $17 / 06 / 200921: 30$ & 728 & & 96 & & 0,8 & & 95 & & 18,9 & & 0 & \\
\hline $17 / 06 / 200922.30$ & 728 & & 97,3 & & 0.2 & & $\infty$ & & 18.4 & & 0 & \\
\hline $17 / 06 / 200923-30$ & 728 & & 98.6 & & 0 & & 0 & & 18,1 & & 0 & \\
\hline $20 / 06 / 200900-30$ & 731 & & 95.4 & & 0 & & 0 & & 17,7 & & 0 & \\
\hline 20066200901,30 & 731 & & 96.6 & & 0 & & 0 & & 17,6 & & 0 & \\
\hline $20106 / 20090230$ & 730 & & 97,4 & & 0 & & 0 & & 17,5 & & 0 & \\
\hline $20106 / 20090330$ & 730 & & 97,4 & & 0 & & 0 & & 17.6 & & 0 & \\
\hline 2000620090430 & 730 & & 98,1 & & 0 & & 0 & & 17,4 & & 0 & \\
\hline $20106 / 2009$ 05 30 & 730 & & $\$ 8,6$ & & 0 & & 0 & & 17,4 & & 0 & \\
\hline $20106 / 20090630$ & 731 & & 98,7 & & 0 & & 0 & & 17,3 & & 0 & \\
\hline $2006 / 2000$ 07:30 & 731 & & 97,5 & & 0 & & 0 & & 17,7 & & 0 & \\
\hline $20106 / 20090830$ & 732 & & 90,5 & & 0 & & 0 & & 19,5 & & 0 & \\
\hline 2000620090930 & 734 & & 7.3 & & 0 & & 0 & & 22.4 & & 0 & \\
\hline 2006200091030 & 734 & & 71,3 & & 0 & & 0 & & 23,4 & & 0 & \\
\hline $20106 / 20091130$ & 733 & & 63,7 & & 0 & & 87 & & 25.4 & & 0 & \\
\hline
\end{tabular}




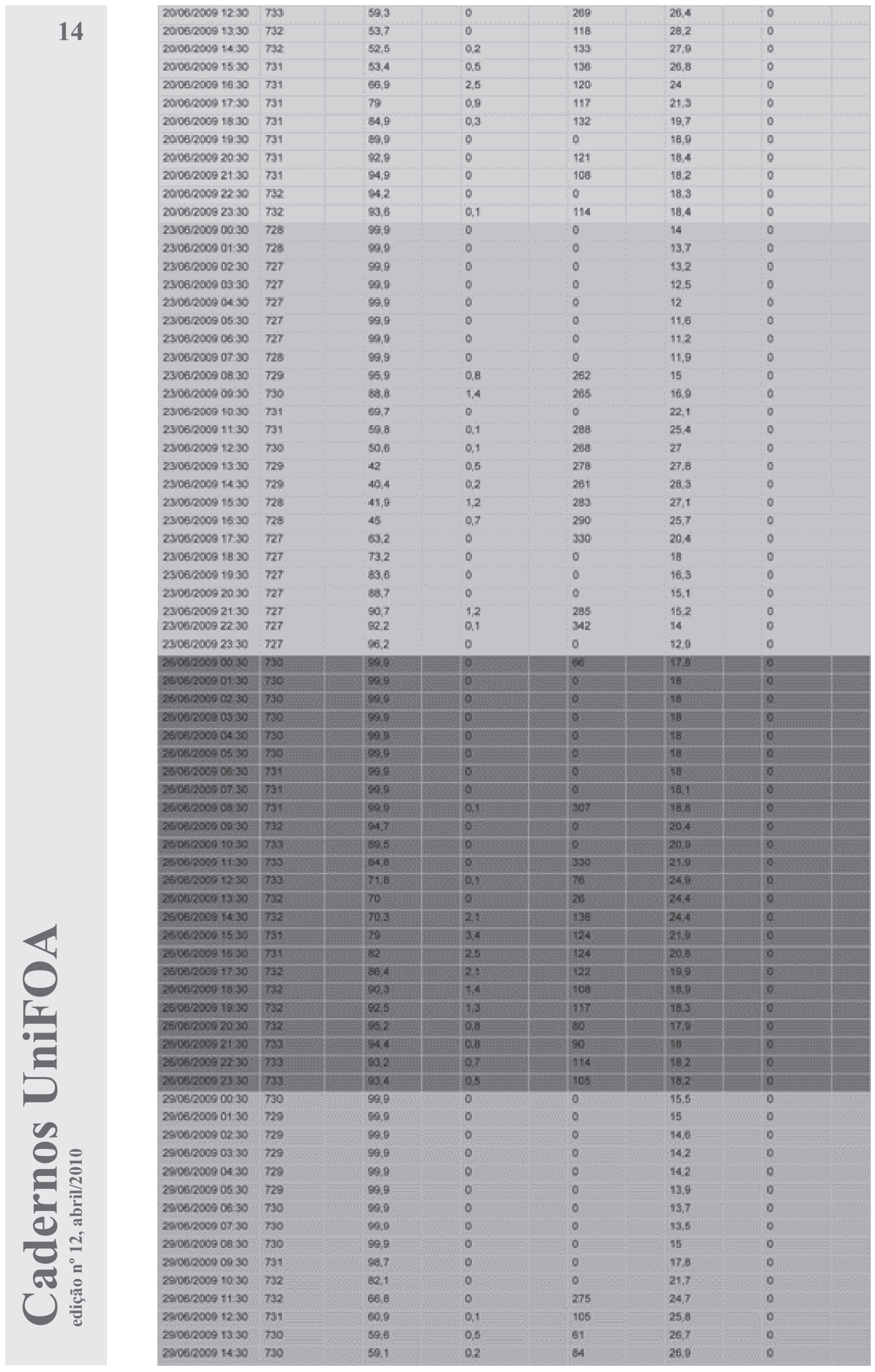




\begin{tabular}{|c|c|c|c|c|c|c|}
\hline $29106200915: 30$ & 730 & 59,5 & 0 & 0 & 27 & 0 \\
\hline $2906 / 20091630$ & 729 & 61,4 & 0 & 30 & 26,4 & 0 \\
\hline 29062009 17:30 & 728 & 72.7 & 0.1 & 105 & 22.7 & 0 \\
\hline $29,06 / 200918-30$ & 728 & 81,4 & 0 & 84 & 20,7 & 0 \\
\hline 290620091230 & 728 & 88.5 & 0 & 0 & 19.6 & 0 \\
\hline 2910620092030 & 728 & 94 & 0 & 0 & 18,4 & 0 \\
\hline $2906 / 200921: 30$ & 728 & 99.3 & 0 & 0 & 17,2 & 0 \\
\hline 2506200092230 & 728 & 20.9 & 0 & 0 & 16.6 & 0 \\
\hline $29106 / 20092330$ & 727 & 99,9 & 0 & 0 & 15,9 & 0 \\
\hline $0307 / 20090030$ & 729 & 82,9 & 0 & 0 & 18.9 & 0 \\
\hline 0s0riac09 01:30 & 729 & 86 & 0 & 0 & 18.5 & 0 \\
\hline $0307 / 20090230$ & 729 & 89,2 & 0 & 0 & 18,3 & 0 \\
\hline $03,07 / 2000903,30$ & 729 & 91.7 & 0 & 0 & 18.2 & 0.2 \\
\hline 0907120090630 & 728 & 96,3 & 0 & 0 & 17,5 & 0.2 \\
\hline $03 / 071200905: 30$ & 728 & 99,8 & 0 & 53 & 17,1 & 0 \\
\hline $0907 \pi / 20000630$ & 729 & 99.8 & 0 & 0 & 17,2 & 0 \\
\hline 080712009 or:30 & 729 & 99,8 & 0 & 0 & 17,8 & 0 \\
\hline $03 / 07 / 20090830$ & 730 & 96,8 & 0.2 & 72 & 18,5 & 0 \\
\hline $0307 / 20090030$ & 731 & 94,4 & 0.3 & 76 & 20 & 0.2 \\
\hline 0s:07i2009 10.30 & 735 & 99,9 & 0.2 & 105 & 21,2 & 0 \\
\hline $03 / 07 i 200911: 30$ & 731 & 82,3 & 1 & 70 & 22,4 & 0 \\
\hline $0307 r 200912.30$ & 731 & 73.7 & 0.9 & 84 & 24 & 0 \\
\hline $03107 / 260913: 30$ & 730 & 732 & 0.3 & 65 & 23,6 & 0 \\
\hline $0907 / 2000914,30$ & 729 & 80 & 0,3 & 91 & 22,4 & 0 \\
\hline 030712000915.30 & 729 & 81.8 & 0.6 & 104 & 22.1 & 0 \\
\hline $03 / 07 / 200916.30$ & 729 & 85 & 1,9 & 119 & 21,1 & 0 \\
\hline $03 / 0712000217,30$ & 729 & 90.5 & 1.8 & 117 & 20.2 & 0 \\
\hline $0307 r 2009$ t8 30 & 730 & 93,6 & 0,1 & 63 & 19.7 & 0 \\
\hline $03,07 / 200918: 30$ & 729 & 96 & 1 & 82 & $1 \mathrm{a}, \theta$ & 0.4 \\
\hline $03 / 07 / 20092030$ & 730 & 99.9 & 1.1 & 100 & 18.5 & 1 \\
\hline $0807 \pi 20092130$ & 730 & 90,8 & 0,3 & 97 & 18,3 & 2 \\
\hline $03 / 07 i 20092230$ & 730 & 99,8 & 0.2 & 48 & 18,1 & 0,4 \\
\hline $03 / 07 / 20092330$ & 729 & 99.9 & 0.5 & 78 & 18 & 0.4 \\
\hline$1 6 0 7 \longdiv { 2 0 0 9 0 0 3 0 }$ & 733 & 97.2 & 0 & 0 & 15.9 & 0 \\
\hline 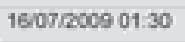 & 733 & 96,6 & 0 & 0 & 16,2 & 0 \\
\hline 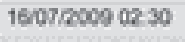 & 732 & 97.3 & 0 & 0 & 16.1 & 0 \\
\hline 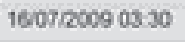 & 732 & 90.2 & 0 & 0 & 15,7 & 0 \\
\hline $16 / 07 / 200904: 30$ & 732 & 99,9 & 0 & 0 & 14,8 & 0 \\
\hline $16 / 07 / 200905: 30$ & 732 & 90.9 & 0 & 0 & 14,4 & 0 \\
\hline 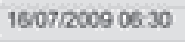 & 732 & 99,9 & 0 & 0 & 14 & 0 \\
\hline $16 / 07 / 200907,30$ & 732 & 99,9 & 0 & 0 & 14,6 & 0 \\
\hline $1607 / 20090830$ & 734 & 93 & 0 & 25 & 19.2 & 0 \\
\hline$1 6 1 0 7 \longdiv { 2 0 0 9 } 0 9 2 3 0$ & 734 & 79,7 & 0.2 & 90 & 21,2 & 0 \\
\hline $16 / 07 / 200910-30$ & 736 & 71,2 & 0,4 & 105 & 23,2 & 0 \\
\hline $16 / 07 / 200911.30$ & 734 & 64,8 & 0.6 & 92 & 24,6 & 0 \\
\hline $16107 / 20091230$ & 733 & 63,4 & 0,9 & 105 & 25 & 0 \\
\hline $16 / 07 / 200913.30$ & 732 & 61,1 & 2 & 104 & 25,8 & 0 \\
\hline $16 / 07 / 2000914: 30$ & 732 & 58.9 & 1 & 102 & 26.4 & 0 \\
\hline $16,07 / 200915: 30$ & 732 & 60,1 & 0,5 & 95 & 25,5 & 0 \\
\hline $16 / 07 / 200916: 30$ & 731 & 60 & 0,5 & 71 & 25,4 & 0 \\
\hline $16 / 07 / 2009$ 17:30 & 731 & 68,6 & 0.2 & 76 & 22,7 & 0 \\
\hline $16 / 07 / 200918: 30$ & 731 & 73,8 & 0 & 0 & 20,6 & 0 \\
\hline $16 / 07 / 200919: 30$ & 732 & 80,4 & 0 & 0 & 19,3 & 0 \\
\hline $16 / 07 / 200920: 30$ & 731 & 87,8 & 0 & 0 & 18,1 & 0 \\
\hline $16 / 07 / 200921: 30$ & 731 & 93,9 & 0 & 0 & 17,2 & 0 \\
\hline $16 / 07 / 200922: 30$ & 731 & 98,3 & 0 & 0 & 16,5 & 0 \\
\hline $16 / 07 / 200923: 30$ & 732 & 99,9 & 0 & 0 & 16,3 & 0 \\
\hline $19 / 07 / 200900: 30$ & 729 & 99,9 & 1,2 & 68 & 19 & 0 \\
\hline $19 / 07 / 200901: 30$ & 729 & 99,9 & 0,9 & 73 & 19 & 0 \\
\hline $19 / 07 / 200902: 30$ & 728 & 99.9 & 0 & 0 & 18,8 & 1.2 \\
\hline $19 / 07 / 200903: 30$ & 728 & 92.9 & 0 & 0 & 18.7 & 0.2 \\
\hline $19 / 07 / 200904: 30$ & 728 & 99.9 & 0 & 0 & 18.6 & 0 \\
\hline 19/07/2009 05:30 & 728 & 99.9 & 0,6 & 113 & 18.5 & 1,2 \\
\hline $19 / 07 / 200906: 30$ & 728 & 99.9 & 0,9 & 116 & 18,2 & 1.2 \\
\hline $19 / 07 / 200907: 30$ & 728 & 99.9 & 0,9 & 95 & 18 & 0.2 \\
\hline $19 / 07 / 200906: 30$ & 729 & 99.9 & 1,7 & 84 & 18,1 & 0 \\
\hline $1907 / 200909: 30$ & 729 & 99.4 & 1,7 & 99 & 18.6 & 0 \\
\hline $19 / 07 / 200910: 30$ & 729 & 96.5 & 0,7 & 89 & 18.7 & 0 \\
\hline $19007 / 200911: 30$ & 729 & 95,6 & 1,5 & 80 & 18,8 & 0 \\
\hline $19 / 07 / 200912: 30$ & 729 & 93,1 & 1,9 & 76 & 19,1 & 0 \\
\hline $18 / 07 / 200913: 30$ & 728 & 89,3 & 2,3 & 83 & 20,1 & 0 \\
\hline $19 / 07 / 200914: 30$ & 728 & 89,8 & 2,8 & 90 & 19,6 & 0 \\
\hline $18 / 07 / 200915: 30$ & 728 & 92.2 & 2,6 & 77 & 18,6 & 0 \\
\hline $19 / 07 / 200916: 30$ & 728 & 92,9 & 2,2 & 85 & 18,4 & 0 \\
\hline
\end{tabular}




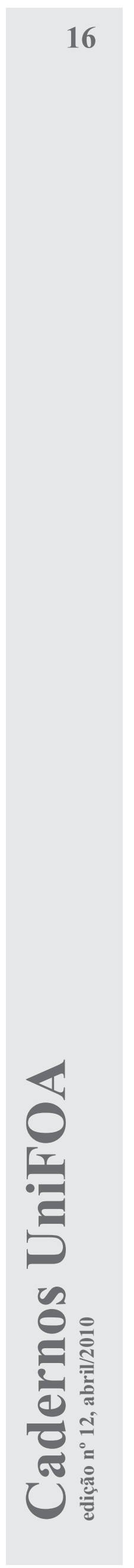

\begin{tabular}{|l|l|l|l|l|l|l|l|}
\hline $19 / 07 / 2009$ & $17: 30$ & 728 & 90,1 & 2,2 & 89 & 17,9 & 0 \\
\hline $19 / 07 / 200918: 30$ & 729 & 90,4 & 1,5 & 86 & 17,4 & 0 \\
\hline $19 / 07 / 200919: 30$ & 729 & 80,3 & 1,1 & 95 & 17,2 & 0 \\
\hline $19 / 07 / 200920: 30$ & 729 & 87,3 & 0,7 & 117 & 17,2 & 0 \\
\hline $19 / 07 / 200921: 30$ & 729 & 86,1 & 1,1 & 116 & 17,1 & 0 \\
\hline $19 / 07 / 200922: 30$ & 730 & 88 & 0,1 & 103 & 16,9 & \\
\hline $19 / 07 / 200923: 30$ & 729 & 90,3 & 0,1 & 57 & 16,7 & 0 \\
\hline
\end{tabular}

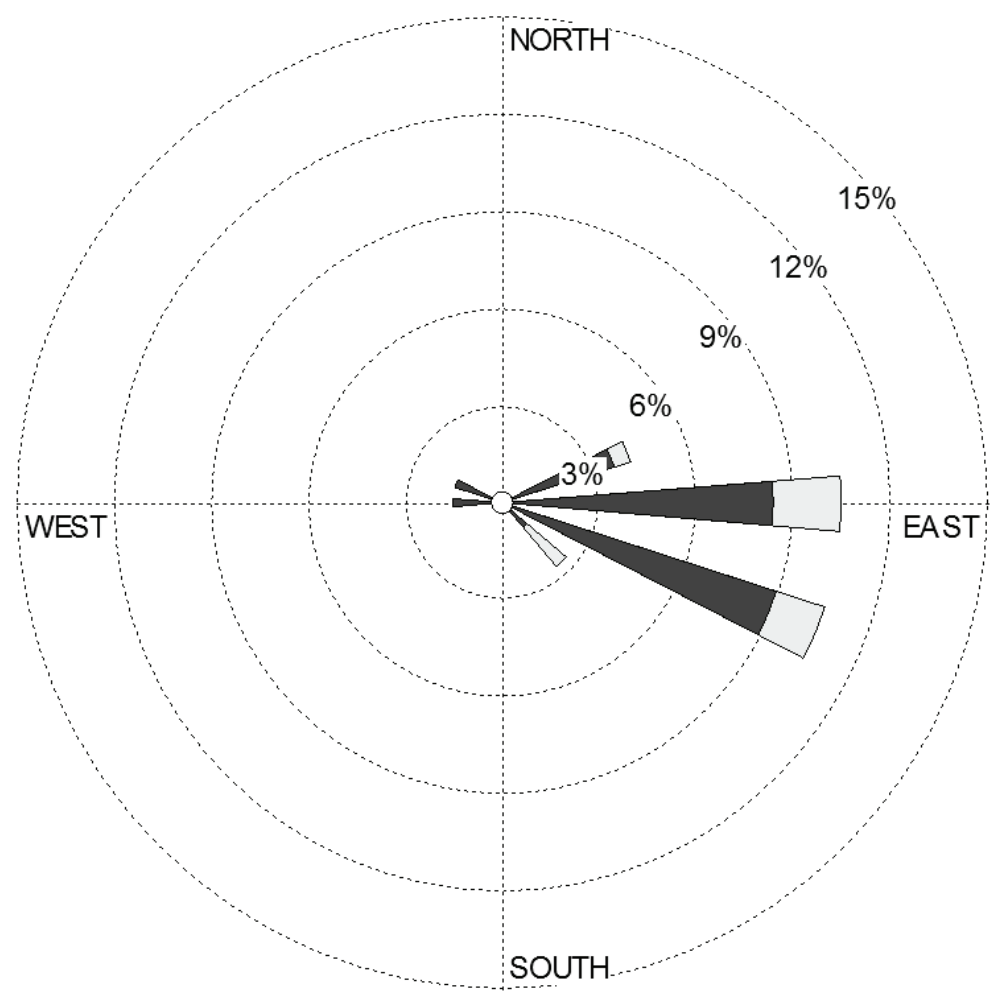

Imagem 01 - Rosa dos Ventos

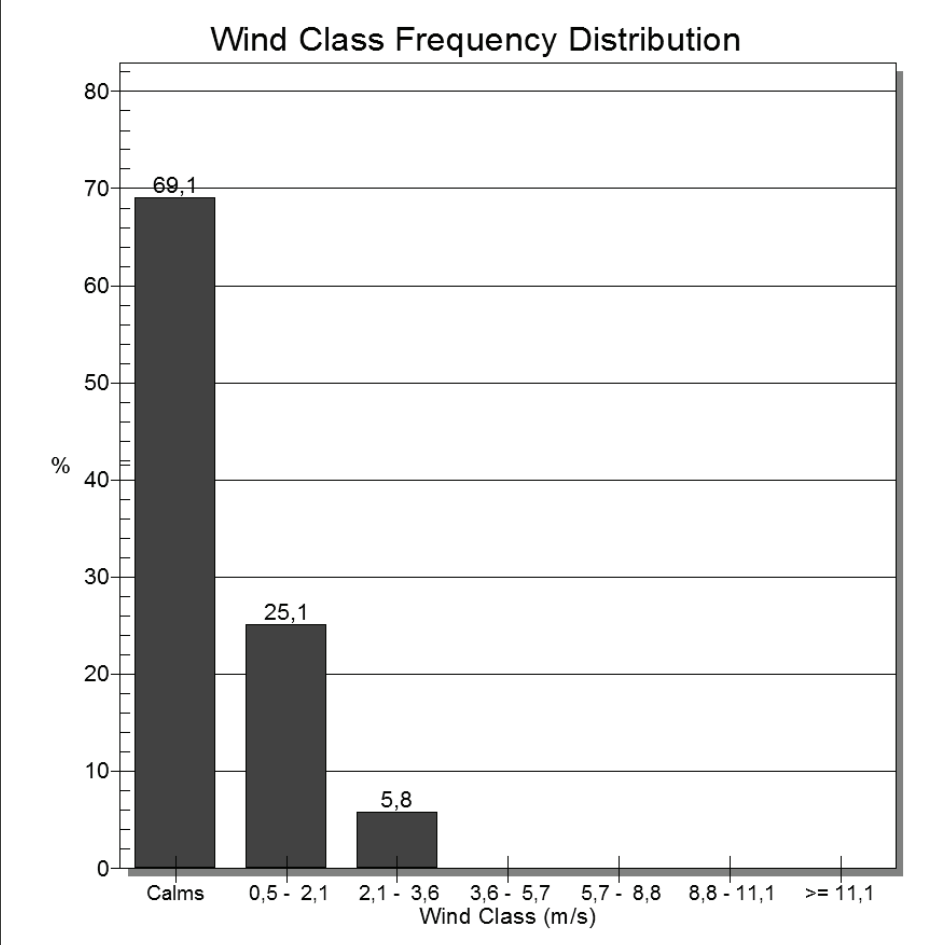

WINC SFEEC

$(\mathrm{m} / \mathrm{s})$

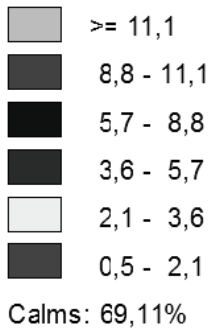




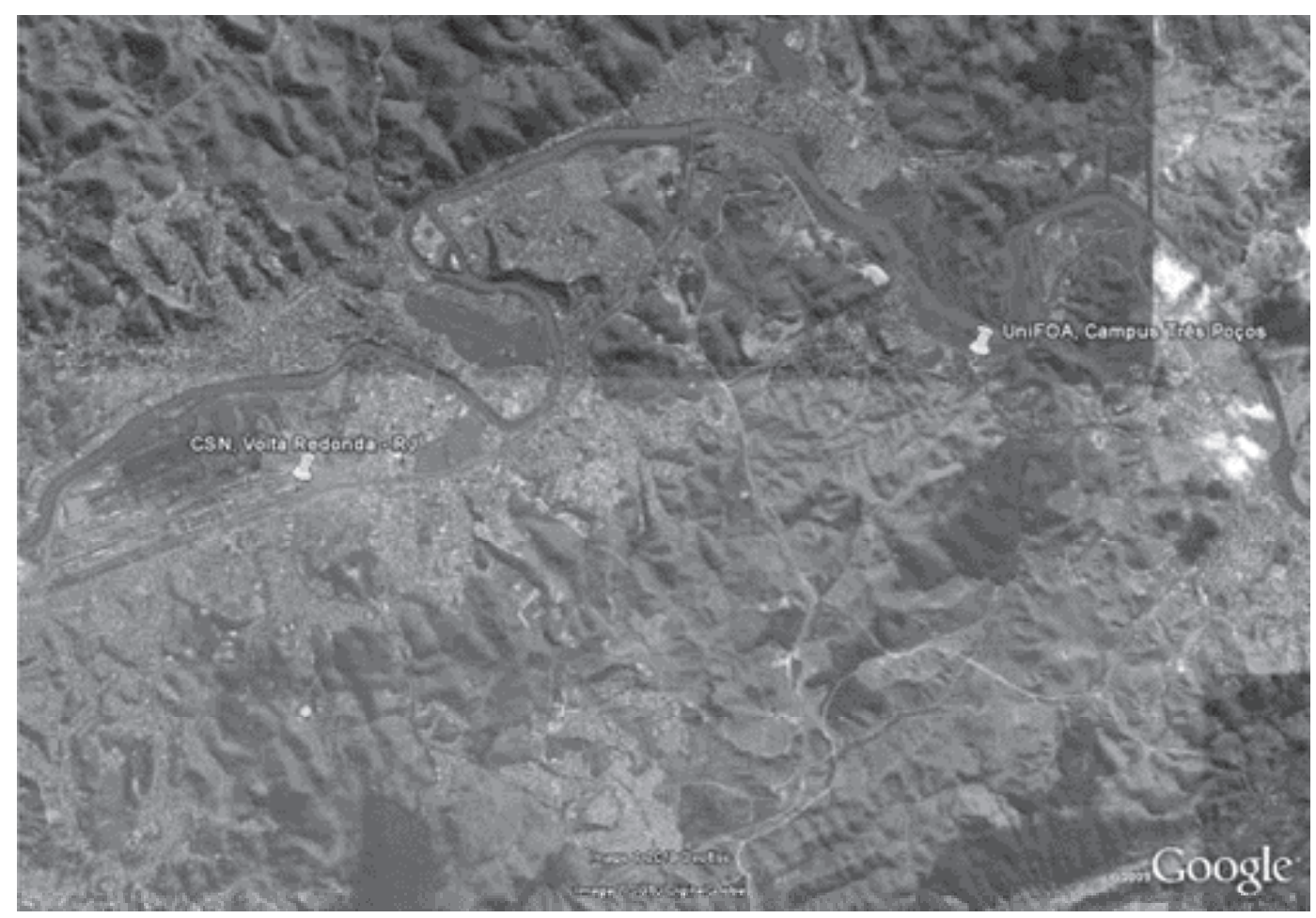

Imagem 03 - Localização

\section{Conclusões}

Após os 4 meses de monitoramento da qualidade do ar no Campus Três Poços, podemos afirmar que:

A qualidade do ar no local da pesquisa, em termos de PTS, não é significativamente influenciada por emissões provenientes de processos industriais;

A qualidade do ar no local da pesquisa, em termos de PTS, é significativamente influenciada por emissões provenientes de fontes móveis (veículos automotores e trens);

Considerando o Padrão Primário definido pela Resolução CONAMA no. 03/90, a média geométrica obtida no período do monitoramento está abaixo do nível considerado como suficiente para afetar a saúde da população local, a saber: $80 \mu \mathrm{g} / \mathrm{m}^{3}$;

Considerando o Padrão Primário definido pela Resolução CONAMA no. 03/90, as concentrações médias obtidas nas 24 horas de cada amostragem, no período do monitoramento, ficaram abaixo do nível considerado como suficiente para afetar a saúde da população local, a saber: $240 \mu \mathrm{g} / \mathrm{m}^{3}$;

Mesmo com a qualidade do ar, em termos de PTS, apresentando valores considerados seguros para a população local, é possível notar que a população flutuante no Campus Três Poços, provoca uma elevação de PTS na atmosfera local de até 4,5 vezes (maior variação observada entre época de provas e época de férias);

Os resultados obtidos podem ser considerados como pontos de maior criticidade, pois conforme os dados meteorológicos dos dias de amostragens, na maioria das vezes o Hi-Vol funcionou em dias sem chuva e com calmaria.

\section{Recomendações Propostas}

Recomendamos que o sistema público de transporte para o bairro Três Poços seja melhorado a fim de reduzir a frota de veículos que se deslocam para o Campus Três Poços em época de aulas;

Recomendamos que seja planejado e realizado um grande programa de "Carona Solidária" nos moldes já implantados em algumas cidades no mundo. Tal programa deverá ser idealizado e gerido pelo UniFOA, visando servir de exemplo, seja para o Município de Volta Redonda, seja para outras cidades do Brasil;

Recomendamos que o UniFOA realize uma ampla campanha de conscientização ambiental para toda a comunidade acadêmica, circunvizinhanças e Poder Público, visando incentivar debates sobre: "Ampliando a 
qualidade de vida em nossa cidade: Como privilegiar o transporte público em detrimento do transporte particular?"

\section{Bibliografia}

1. Fundação Estadual de Engenharia do Meio Ambiente - FEEMA. Perfil Ambiental de Volta Redonda. Rio de Janeiro: FEEMARJ. 1992.

2. Prefeitura Municipal de Volta Redonda Instituto de Pesquisa e Planejamento Urbano de Volta Redonda. Informações para o Plano Diretor - Coleção Cadernos de Planejamento. V. 1. Volta Redonda: IPPU-VR. 1994.

3. Prefeitura Municipal de Volta Redonda Instituto de Pesquisa e Planejamento Urbano de Volta Redonda. Plano Diretor Participativo. V. 1, 2 e 3. Volta Redonda: IPPU-VR. 2008.

4. Resolução CONAMA 18/86: "Dispõe sobre a Instituição do Programa de Controle da Poluição do Ar por Veículos Automotores PROCONVE."

5. Resolução CONAMA 05/89: "Institui o Programa Nacional de Controle de Qualidade do Ar - "PRONAR", e dá outras providências."

6. Resolução CONAMA 03/90; “Estabelece padrões de qualidade do ar e amplia o número de poluentes atmosféricos passíveis de monitoramento e controle."

7. Resolução CONAMA 07/93: "Estabelece padrões de emissão para veículos em circulação."

8. Resolução CONAMA 08/93: "Estabelece limites máximos de emissão de poluentes para motores destinados a veículos pesados novos, nacionais e importados."

9. Resolução CONAMA 16/93: "Ratifica limites de emissão de poluentes por veículos automotores e determina a republicação de Resoluções do CONAMA.“
10. Resolução CONAMA 241/98: "Estabelece prazos para atendimento aos limites de emissão para carros importados."

11. Resolução CONAMA 242/98: "Estabelece limite para emissão de material particulado por veículos."

12. Pollution Prevention and Abatement Handbook, World Bank, World Bank Group - 1998.

13. ENERGÉtICA. Manual de Operação do Amostrador de Partículas Totais em Suspensão. Rio de Janeiro. 2003. 
9. Anexos

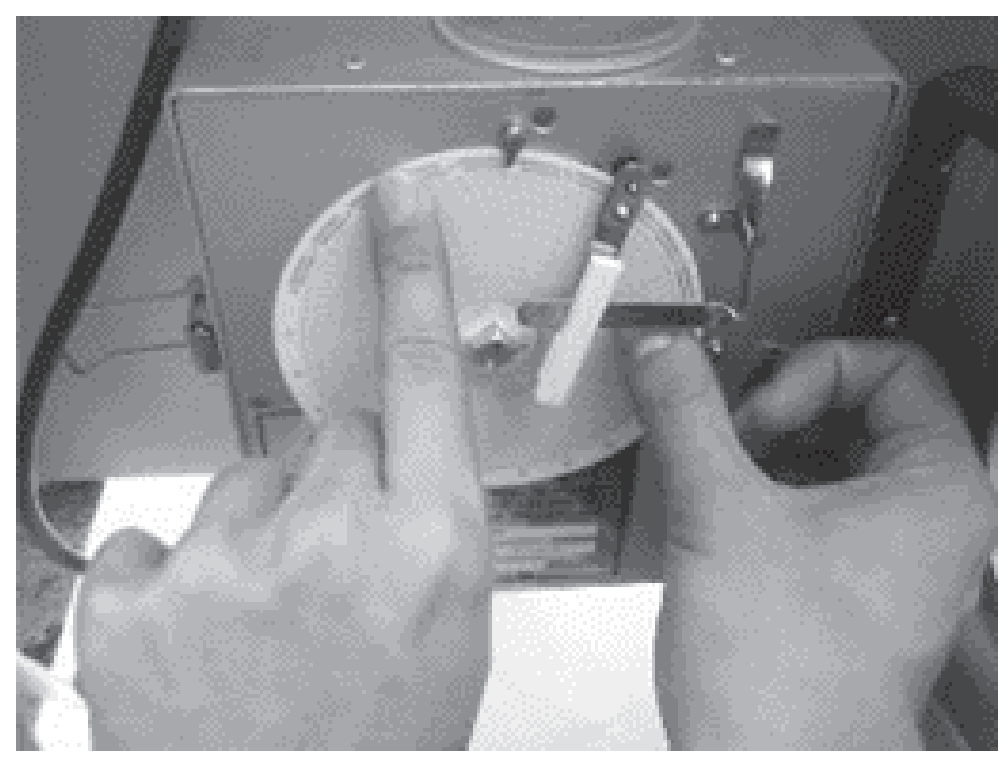

Medidor de Vazão

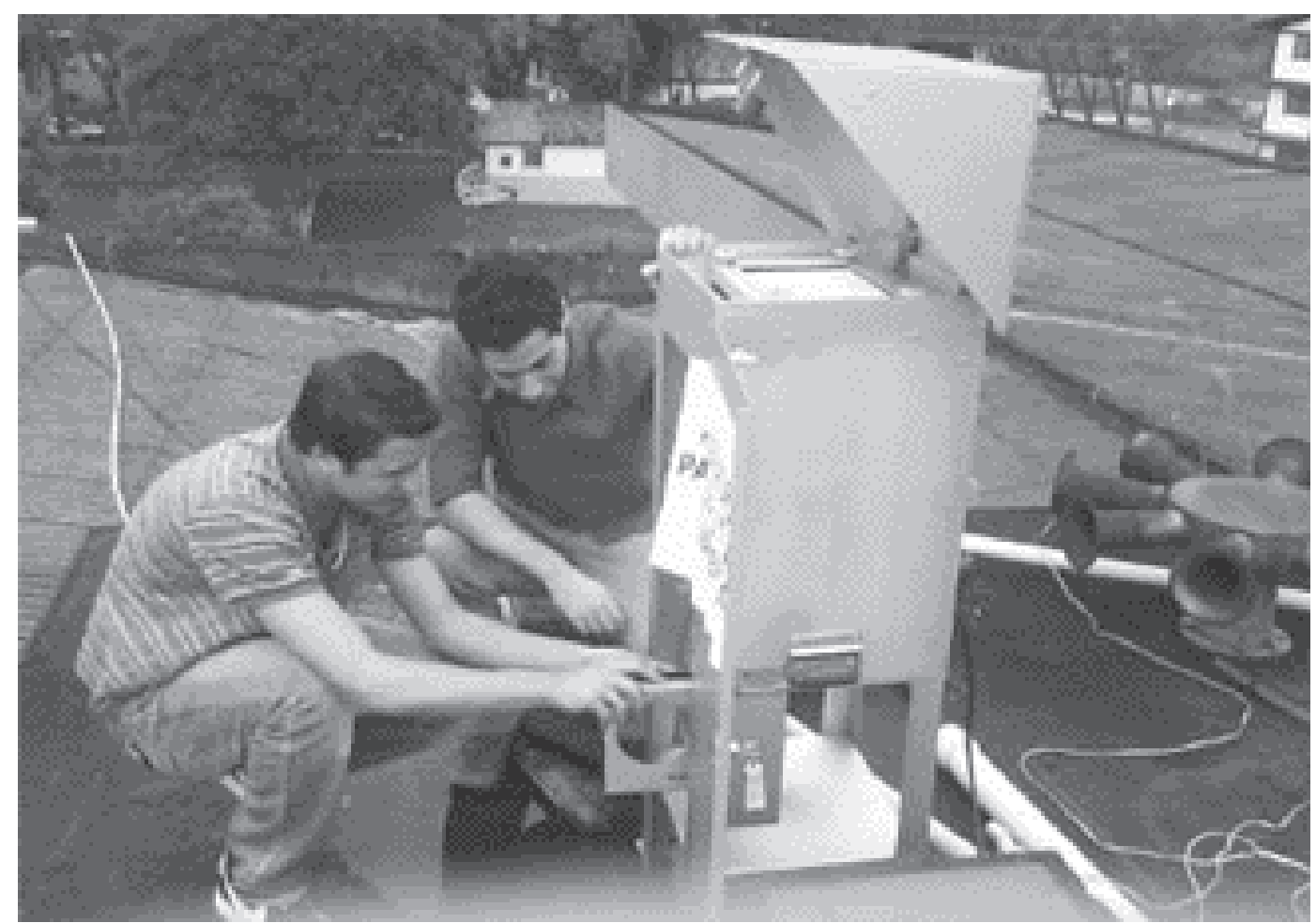

Localização e operação do HI-VOL

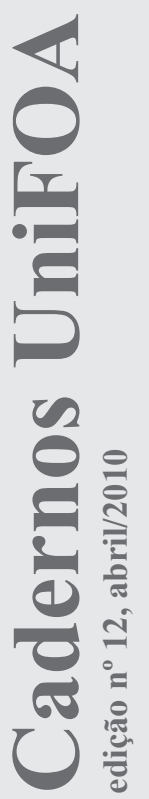



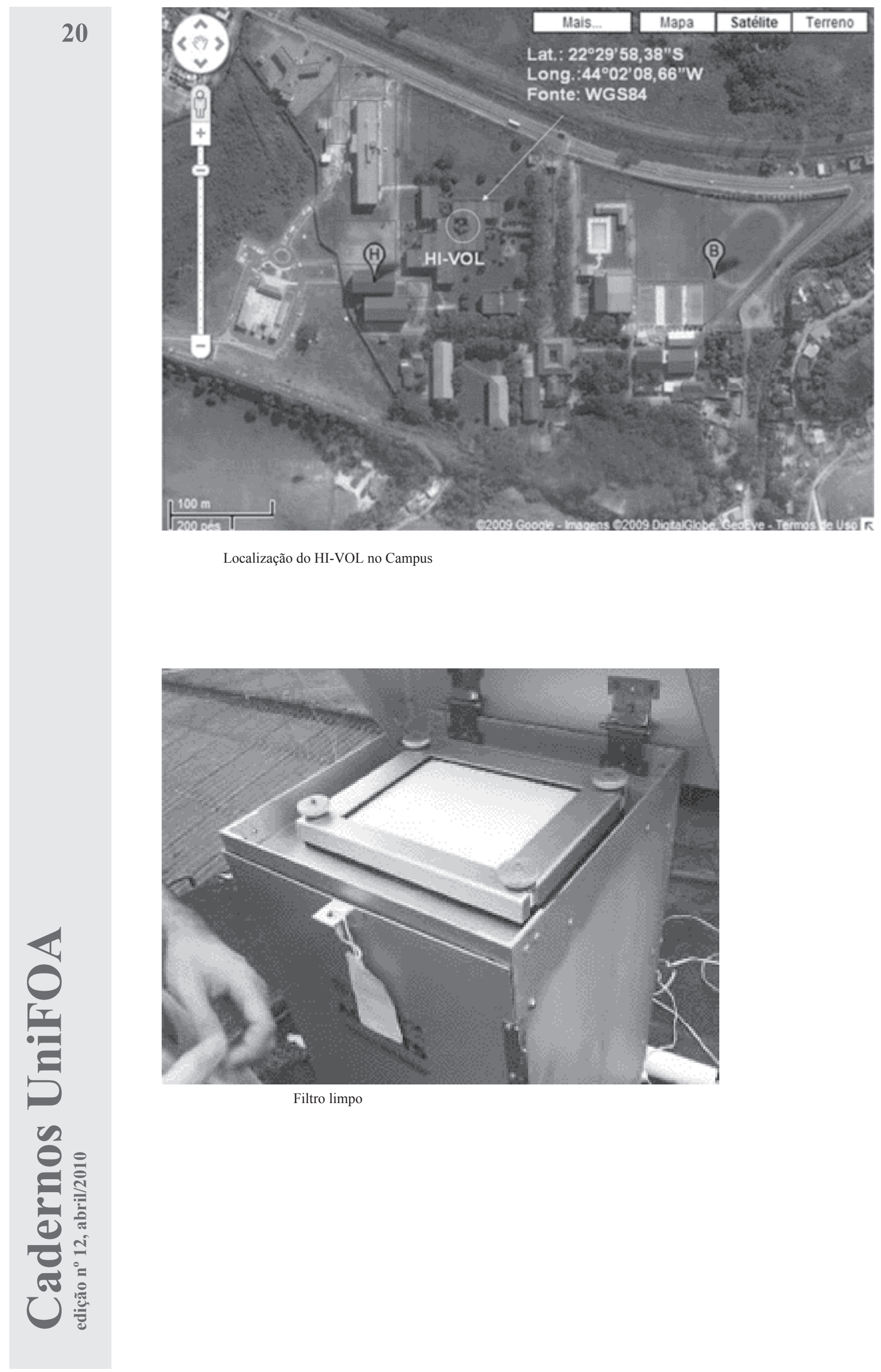

Localização do HI-VOL no Campus

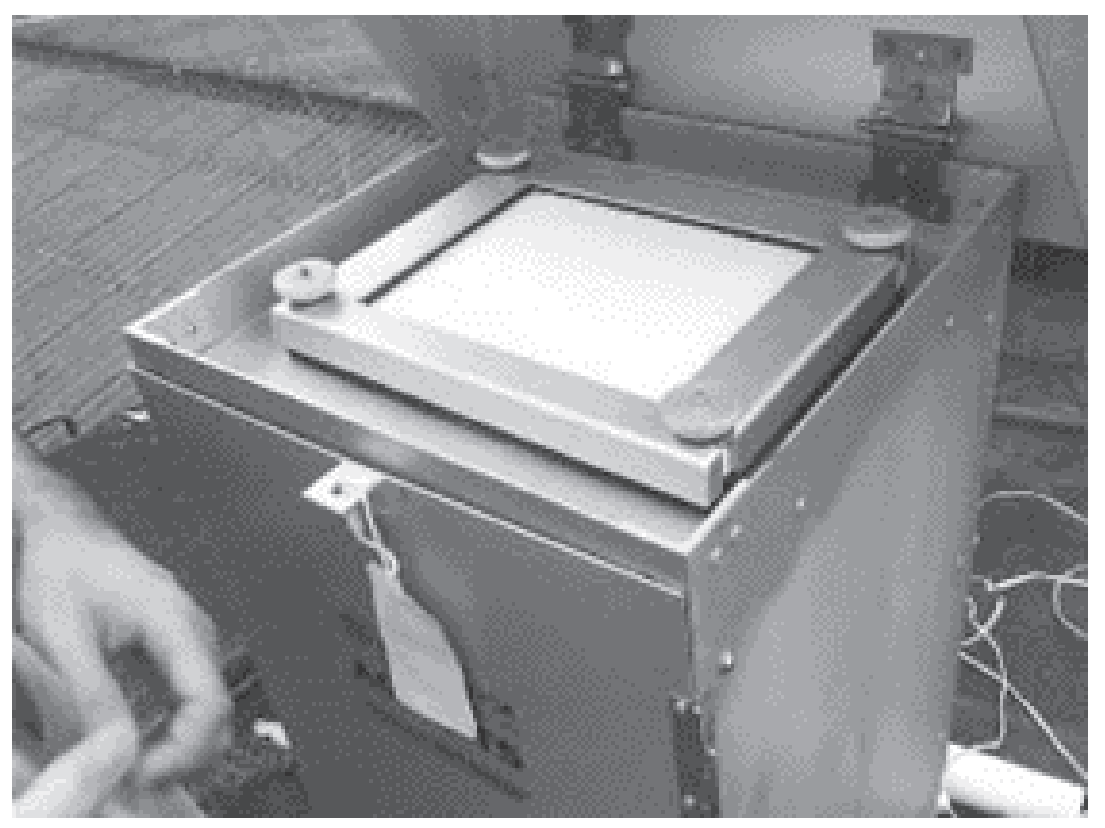

Filtro limpo 


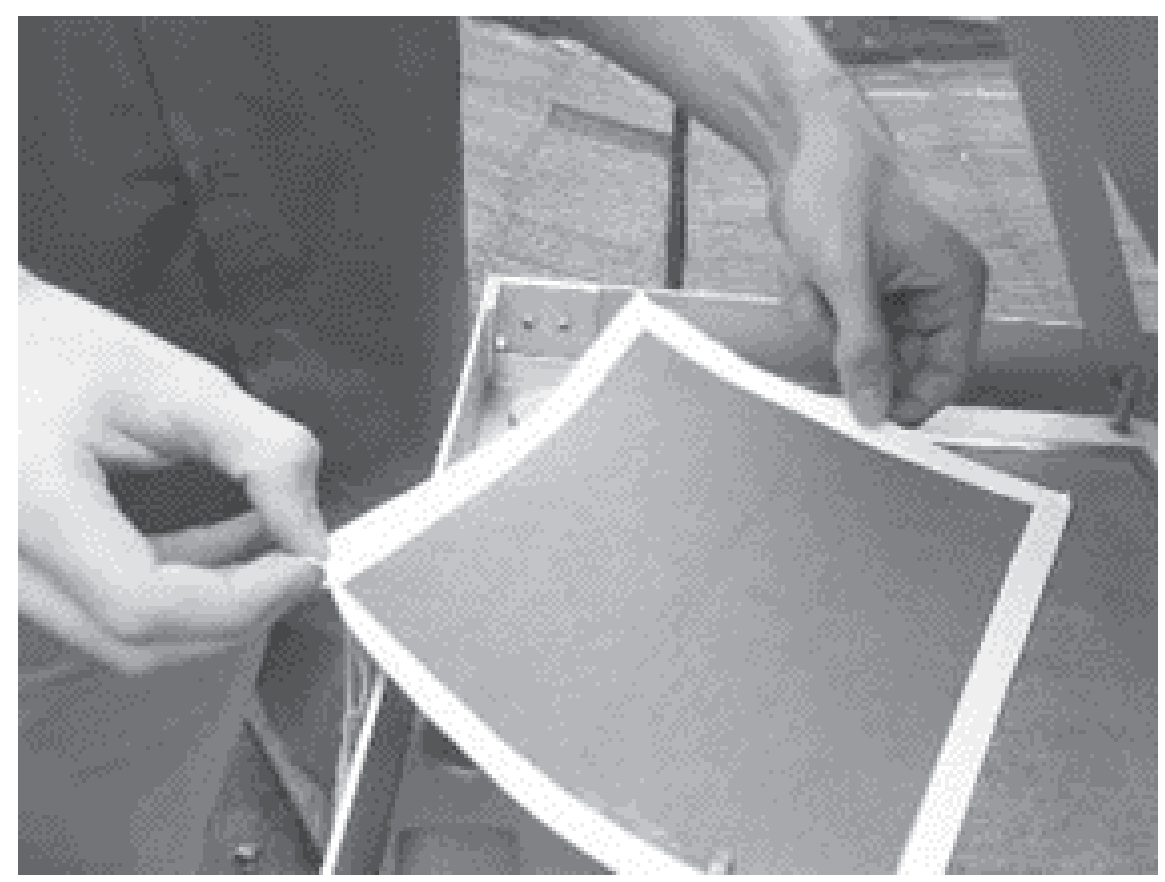

Filtro após 24 hrs de amostragem

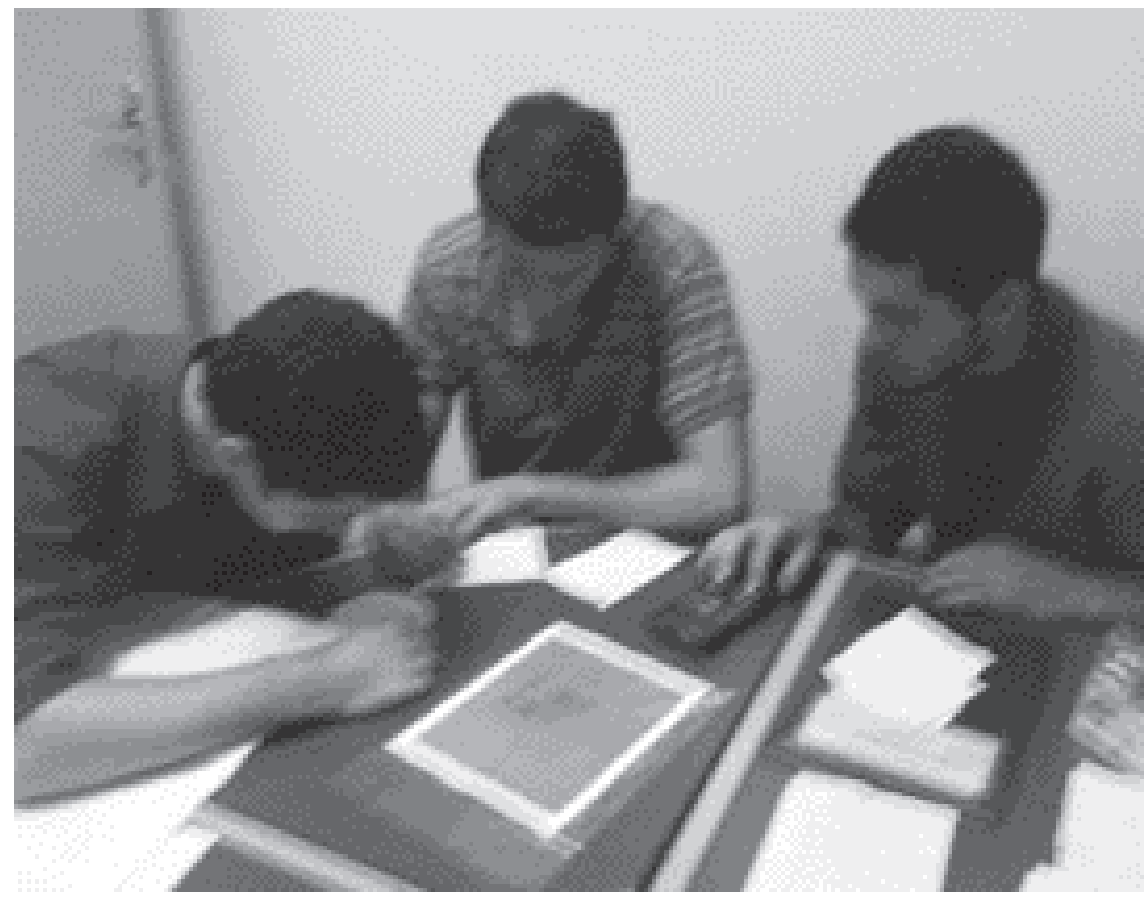

Cálculo das amostragens

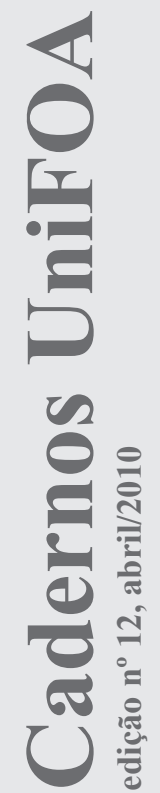




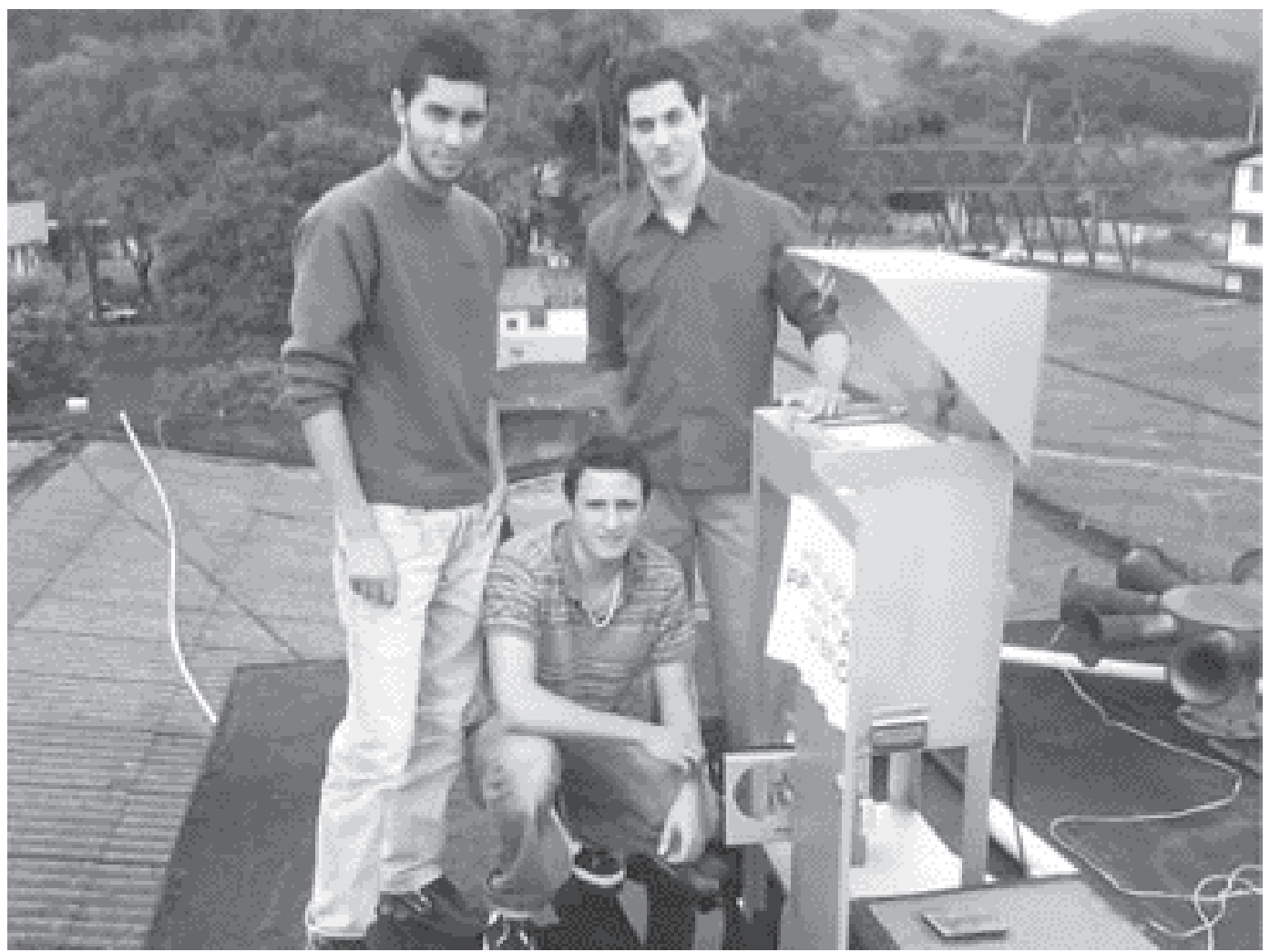

Autores do Projeto

Endereço para Correspondência:

Mateus Nascimento Mariano

mateusnmariano@hotmail.com

Rua Coronel José Mendes Bernardes, n²35,

Jardim Paineiras - Itatiaia/RJ

CEP: 27580-000

(24) 33523821 - (24) 98373216

Informações bibliográficas:

Conforme a NBR 6023:2002 da Associação Brasileira de Normas Técnicas (ABNT), este texto científico publicado em periódico eletrônico deve ser citado da seguinte forma: GOMES, Derek; SÁ, Fabrício Barcelos; MARIANO, Mateus Nascimento; VIANNA, Ramon Castro; GOMES, Anderson; ARAUJO, Marcus Vinicius F. de; SOARES, Rosana GOMES, Derek, SA, Fabricio Barclos, MARIANO, Mateus Nascimento, VIANNA, Ramon Castro, GOMES, Anderson, ARAUJO, Marcus Vhicius F. de, SOARES, Rosana A. Ravaglia. Monitoramento da qualidade do ar no campus Três Poços em termos de partículas totais em suspensão. Cadernos UniFOA. Volta Redonda, ano V, n. 12 , abril 2010.
Disponível em: < $\mathrm{http}: / /$ www.unifoa.edu.br/cadernos/edicao/12/11.pdf $>$ 